\section{O nível de ansiedade traço influencia a percepção de fadiga e bem-estar após diferentes intensidades de exercício físico?}

\section{Does the trait-anxiety level influence the perception of fatigue and well being after different intensities of physical exercise?}

Rafael Eduardo E. P. Chagas Miranda ${ }^{1,2}$

Espedito Pereira da Silva Neto ${ }^{2}$

Marco Túlio De Mello ${ }^{2,3}$

Hanna Karen M. Antunes ${ }^{1,2,4}$

\section{Resumo}

Para investigar se diferentes níveis de ansiedade traço influenciam na percepção da fadiga e bem -estar após exercício físico de moderada e alta intensidade, 26 jovens saudáveis e ativos (idade = $23,23 \pm 3,61$ anos; estatura $=1,75 \pm 0,07 \mathrm{~m}$; massa corporal $=73,26 \pm 8,92 \mathrm{~kg}$ ), foram recrutados Os voluntários foram distribuídos em dois grupos em relação ao nível de ansiedade traço (Grupo ansiedade traço baixa $-G_{\text {ATB }}$ e grupo ansiedade traço media $-G_{\text {ATM }}$ ) realizando os seguintes procedimentos experimentais em diferentes dias utilizando um cicloergômetro: a) carga progressiva até exaustão máxima (TEmax), b) teste em carga retangular na intensidade do limiar ventilatório-I (LV-I) por 30 minutos. Utilizando um modelo de medidas repetidas, foram utilizados questionários para avaliar ansiedade e bem-estar em diferentes momentos (antes, imediatamente após, 30 e 60 minutos após o exercício físico). Os resultados mostraram diminuição significativa dos níveis de ansiedade após 60 minutos. do protocolo intenso (TEmax) em ambos os grupos. Já em relação ao bem-estar, houve aumento significativo na dimensão distresse psicológico após TEmax, apenas para o grupo de ansiedade traço baixa $\left(\mathrm{G}_{\mathrm{ATB}}\right)$. Já para fadiga houve aumento imediato para ambos os grupos estudados após realização de TEmax. Nossos resultados sugerem que independente da ansiedade traço (entre baixa ou média) os jovens responderam de maneira semelhante ao exercício físico de moderada e alta intensidade em relação ao bem-estar e ansiedade, sendo o exercício físico de alta intensidade mais benéfico para estes jovens com efeito ansiolítico em ambos os grupos além de proporcionar manutenção da percepção de bem-estar.

\section{Palavras-chave}

Humor; Atividade Física; Teste de Esforço; Exaustão.

\begin{abstract}
The aim of this study was to investigate if different trait-anxiety levels influences the perception of psychobiological aspects as fatigue and well being after moderate and high intensity physical exercise. Twenty-six health and active male youths participated in the study (age $=23.23 \pm 3.61 \mathrm{yr}$., height $=$ $1.75 \pm 0.07 \mathrm{~m}$, body mass $=73.26 \pm 8.92 \mathrm{~kg}$ ). The volunteers were divided into two trait-anxiety groups (low trait-anxiety - $G_{A T B}$ and medium trait-anxiety - $G_{A T M}$ ), performing the follow experimental procedures on different days using a cycle ergometer: a) progressive cycling to voluntary exhaustion; $b$ ) a moderate-intensity constant load test on ventilatory threshold-I(VTI-30) intensity. Using a repeated measures model, questionnaires were used to assess anxiety, and well being at various time points (before exercise, immediately after exercise, and, 30, 60 minutes after exercise). Results showed decrease in anxiety level after high intensity exercise (TEmax) for both groups. In relation to well being, the psychological distress dimension was increased after TEmax only for $G_{A T B}$ group. Additionally, the fatigue score was increased for both groups after TEmax protocol. Doesn't matter the trait-anxiety level (between low and medium), young individuals responded in a similar way to moderate and high-intensity physical exercise in relation to well being and anxiety, being the high intensity exercise the better one with anxiolytic effect for both groups besides maintain the well being perception.
\end{abstract}

\section{Keywords}

\section{Mood; Physical Activity; Stress Test; Exaustion.}

Rev Bras Ativ Fis Saúde p. 730-739 DOl:

http://dx.doi.org/10.12820/rbafs.v.18n6p730

1 Programa de Pós Graduação Interdisciplinar em Ciências da Saúde, Universidade Federal de São Paulo- Campus Baixada Santista (Unifesp), Santos- SP, Brasil;

2 Centro de Estudos em Psicobiologia e Exercício (CEPE), São Paulo-SP, Brasil;

3 Departamento de Psicobiologia, Universidade Federal de São Paulo- Campus São Paulo (Unifesp), São Paulo-SP, Brasil;

4 Departamento de Biociências, Universidade Federal de São Paulo- Campus Baixada Santista (Unifesp), Santos- SP, Brasil. 


\section{INTRODUÇÃO}

A relação entre exercício físico, ansiedade, e bem-estar tem sido amplamente investigada ${ }^{1,2}$, sendo que em parte, este interesse tem sido motivado pela constatação de que bons níveis de bem-estar estão associados com boa saúde e qualidade de vida ${ }^{3}$.

Em relação a tríade mencionada, grande parte dos estudos tem focado atenção na ansiedade, em particular, no âmbito dos transtornos que a envolve ${ }^{1,2,4}$. Isso se deve aos elevados custos para sociedade em relação ao tratamento desta condição, que pode ser minimizado por meio de intervenção precoce apropriada ${ }^{5}$.

No entanto, sabe-se que a ansiedade não significa necessariamente um estado patológico, isto é, trata-se de uma emoção básica do ser humano compreendida como um estado fisiológico, caracterizado por componentes cognitivos, somáticos emocionais e comportamentais ${ }^{6}$, sendo classificada em ansiedade traço e estado.

A ansiedade estado refere-se ao estado emocional transitório enquanto que a ansiedade traço implica em diferença na capacidade interpessoal de resposta a situações estressantes com variações na ansiedade estado dependendo da extensão e do quanto uma situação específica é percebida como perigo ou ameaça individual ${ }^{7}$. O exercício físico por sua vez é considerado uma estratégia não farmacológica que exerce influência direta na ansiedade ${ }^{8}$ e no bem-estar ${ }^{9}$.

Acevedo e Ekkekakis ${ }^{10}$, relatam existir uma relação inversa entre a intensidade da carga e a adesão ao exercício físico e que tal relação parece ser parcialmente mediada pelo afeto (neste racional, sinônimo de bem-estar), ou seja, maiores intensidades podem acarretar situações menos agradáveis ou desagradáveis no afeto, ocasionando menor adesão e aumento do risco de abandono, pois os indivíduos geralmente escolhem o tipo de exercício físico que os fazem se sentir bem.

Em outras palavras, a ansiedade estado se associa inversamente com o afeto positivo $^{11}$, ou seja, diminuições de ansiedade estado são associadas a aumentos de afeto positivo e consequente melhora no bem-estar.

Porém, sabe-se que mudanças na ansiedade estado requerem sessões de exercício físico em moderada a alta intensidade, sendo o estímulo de baixa intensidade insuficiente para promover alterações efetivas na ansiedade estado ${ }^{12}$ necessitando de maiores investigações acerca da relação entre afeto e ansiedade em resposta ao exercício físico.

Visto que a ansiedade estado exerce influência sobre o bem-estar, nenhum estudo até o momento, abordou a relação entre a ansiedade traço (referente à personalidade) e a percepção de aspectos psicobiológicos como fadiga e sensação de bem-estar.

Neste sentido, uma vez que a ansiedade traço influencia o comportamento ${ }^{13} \mathrm{e}$ a fadiga é percebida conscientemente ${ }^{14}$ o objetivo do estudo foi investigar se diferentes níveis de ansiedade traço (referente a personalidade) influenciam na percepção de aspectos psicobiológicos como fadiga e bem-estar após exercício físico de moderada e alta intensidade.

\section{MÉTODOS}

Inicialmente o estudo foi submetido e aprovado pelo Comitê de Ética da Universidade Federal de São Paulo/Hospital São Paulo (\#1629/08). Assim, vinte e seis voluntários, jovens, saudáveis, do gênero masculino (idade= 23,23 $\pm 3,61$ anos; estatura $=174 \pm 7,11 \mathrm{~cm}$; massa corporal $=73,26 \pm 8,92 \mathrm{~kg}$ ) foram selecionados voluntariamente para o estudo baseados nos critérios de inclusão: a) sexo masculino; b) 
idade entre 18 a 30 anos; c) fisicamente ativo; d) sem histórico de qualquer doença psiquiátrica; e) sem qualquer limitação física ou presença de lesão muscular; f) não fumantes; g) sem alterações no padrão do eletrocardiograma de repouso e esforço.

\section{Desenho Experimental}

Cada voluntário foi ao laboratório por três vezes sendo submetido a duas sessões de exercício físico com intervalo de uma semana. A primeira visita consistiu em assinatura do termo de consentimento livre e esclarecido assim como avaliação da saúde cardiorrespiratória através do eletrocardiograma de repouso e teste ergométrico realizado pelo médico do laboratório.

Uma vez considerados aptos a prática de exercício físico, os voluntários retornaram ao laboratório, após uma semana, para preenchimento do instrumento IDATE-Traço ${ }^{7}$, sendo divididos em dois grupos distintos de acordo com a ansiedade-traço resultante do instrumento, ou seja, $G_{\text {ATB }}$ (grupo ansiedade traço baixa com escore final entre 0 a 30) e $G_{\text {ATM }}$ (grupo ansiedade traço média, com escore final entre 31 a 49), com treze voluntários em cada grupo.

Os critérios para dividir os grupos foram baseados no manual de aplicação do questionário ${ }^{7}$. Os dois grupos $\left(\mathrm{G}_{\mathrm{ATB}}\right.$ e $\left.\mathrm{G}_{\mathrm{ATM}}\right)$, executaram, de maneira semelhante, dois protocolos de exercício físico em moderada e alta intensidade. A ordem de execução dos testes ocorreu de forma aleatória, executado por meio do software online GraphPad Software (http://www.graphpad.com/quickcalcs/).

\section{Procedimentos}

Os dois protocolos de exercício físico foram conduzidos em um cicloergômetro de membro inferior isocinético (Lode, Excalibur Sport 925900, Groningen, Netherlands) com uma cadência de pedalada mínima estipulada em 70 rotações por minuto (RPM). O modo hiperbólico do ergômetro foi acionado, ou seja, a potência gerada em watts foi mantida constante independente do RPM imposto, permitindo desta forma maior segurança em afirmar que a pequena variação do RPM durante os testes em nada impactou na vatagem gerada durante os protocolos executados.

De maneira conjunta, foi realizada uma ergoespirometria, para análise dos gases ventilatórios, determinando as seguintes variáveis respiratórias: consumo pico de oxigênio $\left(\mathrm{VO}_{2 \text { pico }}\right)$, através do sistema metabólico (COSMED modelo Quark PFT - Pulmonary Function Testing - FRC \& DLCO, 4ERGO, Italy), sendo recalibrado para cada teste utilizando concentração de gases conhecida e mascara facial Hans Rudolph ${ }^{\circledR}$ flow-by face mask (Kansas City, MO, EUA).

Durante todos os testes, a monitoração da frequência cardíaca foi realizada por meio de um frequencímetro (Polar ${ }^{\oplus}$, modelo FS1, Kempele, Finland) com intervalos de 5 segundos, assim como a pressão arterial por meio de um estetoscópio duosonic / adulto, e esfigmomanômetro $\mathrm{BD}^{\circledR}$. Os testes foram realizados no mesmo período do dia (9:00 as 12:00) com temperatura ambiente controlada $\left(20-22^{\circ} \mathrm{C}\right)$.

Para cada sessão de exercício físico os participantes responderam aos instrumentos de ansiedade estado e bem-estar nos seguintes momentos; antes, imediatamente, 30 e 60 minutos após o exercício.

\section{Protocolos de Exercício Físico}

- Carga Progressiva até Exaustão Voluntária Máxima (TEmax):

O protocolo adaptado de Denadai ${ }^{15}$ et al., para o teste de carga progressiva até a exaustão voluntária máxima, consistiu em incrementos de 35 watts a cada 2 mi- 
nutos com uma carga inicial de aquecimento fixada em 70 watts. Durante todo o teste os voluntários foram encorajados verbalmente e solicitados a manter $70 \mathrm{rpm}$, sendo o teste encerrado ao atingirem a exaustão voluntária máxima, ou seja, até que o voluntário parasse de se exercitar espontaneamente mesmo sendo encorajado verbalmente ${ }^{16}$, frequência cardíaca acima de $10 \mathrm{~b} \cdot \mathrm{min}^{-1}$ do valor previsto para a idade ${ }^{17}$ e percepção subjetiva de esforço > 18 na escala de Borg. Para a determinação do LV-I foram observados os critérios descritos por Wasserman ${ }^{18}$.

- Teste em carga retangular na intensidade do Limiar Aeróbio (Limiar ventilatório-I [LV-I]) por 30 minutos (LVI-30):

Este protocolo foi executado com carga constante e contínua, correspondente a intensidade do limiar ventilatório I. Durante todo o teste os voluntários foram encorajados verbalmente e solicitados a manter uma cadência mínima de $70 \mathrm{rpm}$, sendo encerrado o teste quando os voluntários atingiram o tempo de 30 minutos, pré-estabelecido. Todos os voluntários completaram o tempo estipulado e mantiveram a cadência mínima.

\section{Instrumentos Aplicados}

Para cada protocolo de exercício físico, os voluntários responderam a instrumentos que avaliaram o afeto ou bem-estar e ansiedade nos seguintes momentos: basal, imediatamente, 30 e 60 minutos após o exercício.

- Escala Subjetiva de Experiência em Exercício- SEES

A sensação de bem-estar foi quantificada através da Escala Subjetiva de Experiência em Exercício- SEES ${ }^{19,20}$ - Instrumento utilizado para observar respostas afetivas induzidas pelo exercício físico. Trata-se de uma escala tridimensional, que avalia as seguintes dimensões: (a) bem-estar positivo; (b) distresse psicológico e (c) fadiga. A principal questão é: “Como você se sente agora?", a escala é composta por 12 itens graduados em uma escala tipo Likert que varia de 1 a 7 , onde 1 significa nenhum pouco e 7 significa muitíssimo.

\section{- Inventário de Ansiedade Traço-Estado (IDATE)}

A ansiedade foi quantificada através de um Inventário de Ansiedade Traço-Estado (State Trait Anxiety Inventory - STAI) - Este instrumento autoavaliativo está dividido em duas partes: uma avalia a ansiedade-traço (referindo-se a aspectos de personalidade) e a segunda avalia a ansiedade-estado (referindo-se a aspectos sistêmicos do contexto). Cada uma dessas partes é composta de 20 afirmações. Ao responder o questionário, o indivíduo deve levar em consideração uma escala de quatro itens que variam de 1 a 4 , sendo que ESTADO significa como o sujeito se sente no "momento" e TRAÇO como ele "geralmente se sente". O escore de cada parte varia de 20 a 80 pontos, sendo que os escores podem indicar um baixo grau de ansiedade (0-30), um grau mediano de ansiedade (31-49) e um grau elevado de ansiedade (maior ou igual a 50), quanto mais baixo se apresentarem os escores, menor será o grau de ansiedade ${ }^{7,21}$. É importante mencionar que nesse estudo, a parte referente à ansiedade Traço foi aplicada em um único momento (início do estudo), por referir ao traço de personalidade do voluntário.

\section{Análise estatística}

foi aplicado o teste Shapiro Wilk para verificação da normalidade dos dados e Teste de Levene para verificar a homogenidade das variâncias. Os resultados estão 
expressos em média \pm desvio-padrão. Dados foram comparados através de Teste- $t$ de Student para amostras independentes, assim como análise de variância ANOVA two-way (protocolo $\mathrm{x}$ tempo) para medidas repetidas com o teste post-hoc Tukey. Para todas as análises, o nível de significância adotado foi de $\mathrm{p} \leq 0,05$ e o tamanho do efeito $(\eta) \geq 0,30$ e o poder observado $(\omega) \geq 0,80$. Foi utilizado o software STATISTICA 12.0 (StatSoft, Inc., Tulsa, OK, USA).

\section{RESULTADOS}

Os voluntários apresentaram $\mathrm{VO}_{2 \text { pico }}$ de 43,98 \pm 7,55 ml.kg.min ${ }^{-1}$, frequência cardíaca máxima (FCmax) de 187,69 \pm 7,88 bpm e carga atingida no $\mathrm{VO}_{2 \text { pico }}$ de $282,92 \pm 32,50$ watts, sendo caracterizados como fisicamente ativos. Como demonstrado na tabela 1 , os grupos não apresentaram diferença significativa em nenhum parâmetro fisiológico considerado, demonstrando homogeneidade da amostra estudada.

Tabela 1 - Análise descritiva dos parâmetros fisiológicos dividido por grupos.

\begin{tabular}{lccc}
\multicolumn{1}{c}{ Variável } & $\mathrm{G}_{\text {ATB }}$ & $\mathrm{G}_{\text {ATM }}$ & $\mathrm{p}$ \\
\hline $\mathrm{VO}_{2}$ pico $\left(\mathrm{L}_{\mathrm{min}}{ }^{-1}\right)$ & $3,39 \pm 0,65$ & $3,18 \pm 0,58$ & 0,40 \\
\hline $\mathrm{VO}_{2}$ pico $\left(\mathrm{mL} \cdot \mathrm{kg}^{-1} \cdot \mathrm{min}^{-1}\right)$ & $43,28 \pm 5,41$ & $44,68 \pm 9,40$ & 0,65 \\
\hline $\mathrm{FC} \max (\mathrm{bpm})$ & $188,31 \pm 6,90$ & $187,08 \pm 9,00$ & 0,70 \\
\hline $\mathrm{VO}_{2}$ LVI (mL.kg $\left.{ }^{-1} \cdot \mathrm{min}^{-1}\right)$ & $27,53 \pm 5,29$ & $30,23 \pm 6,86$ & 0,27 \\
\hline Carga LVI (watts) & $150,08 \pm 15,90$ & $164,23 \pm 43,78$ & 0,28 \\
\hline Carga máx. (watts) & $285,39 \pm 31,46$ & $280,00 \pm 35,00$ & 0,73 \\
\hline
\end{tabular}

Teste- $t$ de Student para amostras independentes. Não houve diferença estatística entre grupos em nenhuma variável. Dados apresentados em média \pm desvio-padrão. Dados referentes a 13 voluntários em cada grupo.

$\mathrm{Na}$ tabela 2, são apresentados a média dos escores de ansiedade estado dos grupos $\mathrm{G}_{\text {ATB }}$ e $\mathrm{G}_{\text {ATM }}$. Os grupos foram divididos em Ansiedade Baixa (0 a 30) e Ansiedade Média (31 a 49) como mostra a tabela 2 apresentando diferença significativa $(\mathrm{p}=0,00001)$ entre eles.

Tabela 2 - Análise dos resultados referentes ao nível de ansiedade traço.

\begin{tabular}{lccc}
\hline Nível de ansiedade traço & Média \pm Desvio padrão & Valor Mínimo & Valor Máximo \\
\hline$G_{\text {ATB }}$ & $26,08 \pm 3,04^{*}$ & 22,00 & 30,00 \\
\hline$G_{\text {ATM }}$ & $38,92 \pm 4,25$ & 32,00 & 48,00 \\
\hline
\end{tabular}

Teste- $t$ de Student para amostras independentes. * - diferente do $G_{\text {ATM }}$. Resultados significativos para $p \leq 0,05$. Dados apresentados em média \pm desvio-padrão. Dados referentes a 13 voluntários em cada grupo. Legenda: $\mathrm{G}_{\text {Атв- }}$ Grupo ansiedade traço baixa; $\mathrm{G}_{\text {Атм- }}$ Grupo ansiedade traço média.

Em relação aos parâmetros psicobiológicos avaliados, na tabela 3 são apresentados os resultados referentes ao teste de ansiedade (IDATE Estado) para ambos os grupos, nos diferentes momentos, considerando cada protocolo de exercício físico. Para o protocolo de alta intensidade (TEmax) houve diferença significativa no momento 60 minutos em relação à condição basal, tanto no grupo de ansiedade baixa ( $\mathrm{p}=0,03)$, quanto no grupo de ansiedade média $(\mathrm{p}=0,02)$. No protocolo de intensidade moderada (LVI-30), não houve diferença significativa, para nenhum grupo, em relação ao nível de ansiedade nos diferentes momentos considerados. 
Tabela 3 - Resultados de ansiedade estado (IDATE) nos diferentes protocolos de exercício físico e momentos considerados.

\begin{tabular}{|c|c|c|c|c|c|}
\hline & & Basal & Imediatamente Após & 30 minutos & 60 minutos \\
\hline \multirow{4}{*}{ 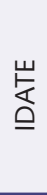 } & $\mathrm{G}_{\mathrm{ATB}} / \mathrm{TEmax}$ & $34,80 \pm 9,16$ & $32,54 \pm 6,74$ & $29,38 \pm 2,84$ & $28,77 \pm 3,35^{a}$ \\
\hline & $\mathrm{G}_{\mathrm{ATB}} / \mathrm{LVI}-30$ & $29,46 \pm 5,90$ & $28,23 \pm 3,63$ & $27,46 \pm 5,75$ & $28,15 \pm 4,28$ \\
\hline & $\mathrm{G}_{\text {ATM }} /$ TEmax & $39,59 \pm 8,05$ & $38,46 \pm 7,09$ & $35,69 \pm 8,20$ & $33,77 \pm 7,35^{a}$ \\
\hline & $\mathrm{G}_{\text {ATM }} / \mathrm{LVI}-30$ & $33,23 \pm 5,10$ & $34,15 \pm 10,09$ & $32,15 \pm 7,06$ & $32,69 \pm 7,27$ \\
\hline
\end{tabular}

ANOVA para medidas repetidas, com post-hoc utilizando Tukey Test. ${ }^{a}$ - diferente do basal. Resultados significativos para $\mathrm{p} \leq 0,05$. Dados apresentados em média \pm desvio-padrão. Legenda: Grupo/ Condição, onde: $\mathrm{G}_{\mathrm{ATB}} / \mathrm{TEmax}$ - Grupo ansiedade traço baixa/Teste de carga progressiva até exaustão máxima; $\mathrm{G}_{\mathrm{ATB}} / \mathrm{LVI}$-30- Grupo ansiedade traço baixa/Teste em carga retangular na intensidade do limiar ventilatório I por 30 minutos; $\mathrm{G}_{\text {ATM }}$ /TEmax- Grupo ansiedade traço média/Teste de carga progressiva até exaustão máxima; $\mathrm{G}_{\mathrm{ATM}} / \mathrm{LVI}$-30- Grupo ansiedade traço média/Teste em carga retangular na intensidade do limiar ventilatório I por 30 minutos.

$\mathrm{Na}$ tabela 4 apresentamos os resultados da Escala Subjetiva de Experiência em Exercício, instrumento utilizado para avaliar o bem-estar para o grupo $G_{\mathrm{ATB}}$ Observamos que quando o grupo $\mathrm{G}_{\text {ATB }}$ executou o protocolo de alta intensidade (TEmax), houve diferença nas dimensões distresse psicológico e fadiga. $\mathrm{Na}$ dimensão distresse psicológico, o momento imediatamente após foi diferente $(p=0,03)$ da condição basal. Já na dimensão fadiga, houve diferença nos momentos imediatamente após $(\mathrm{p}=0,0002) ; 30$ minutos $(\mathrm{p}=0,0004) ; 60$ minutos $(\mathrm{p}=0,04)$, em relação à condição basal. Ainda nesta dimensão, houve diferenças nos momentos 30 minutos $(p=0,007)$ e 60 minutos ( $p=0,0002)$, em relação a condição imediatamente após.

Quando este mesmo grupo realizou o protocolo de moderada intensidade (LVI-30), houve diferença somente para a dimensão fadiga nos momentos $30 \mathrm{mi}$ nutos $(\mathrm{p}=0,025) ; 60$ minutos $(\mathrm{p}=0,05)$ em relação a condição imediatamente após.

Tabela 4 - Resultados de bem estar (SEES) nos diferentes protocolos de exercício físico e momentos considerados para Grupo de Ansiedade Baixa (GATB).

\begin{tabular}{|c|c|c|c|c|c|}
\hline \multicolumn{2}{|l|}{ Variáveis } & \multirow{2}{*}{ Basal } & \multirow{2}{*}{$\begin{array}{l}\text { Imediatamente } \\
\text { Após }\end{array}$} & \multirow{2}{*}{30 minutos } & \multirow{2}{*}{60 minutos } \\
\hline Dimensões & Testes & & & & \\
\hline \multirow{2}{*}{$\begin{array}{l}\text { Bem-estar } \\
\text { Positivo }\end{array}$} & TEmax & $17,69 \pm 3,61$ & $15,92 \pm 3,62$ & $15,77 \pm 3,81$ & $16,38 \pm 4,11$ \\
\hline & LVI-30 & $16,77 \pm 3,70$ & $16,92 \pm 3,45$ & $17,38 \pm 3,99$ & $17,23 \pm 4,13$ \\
\hline \multirow{2}{*}{$\begin{array}{l}\text { Distresse } \\
\text { Psicológico }\end{array}$} & TEmax & $4,54 \pm 0,88$ & $6,77 \pm 3,88^{a}$ & $5,23 \pm 1,64$ & $5,08 \pm 1,61$ \\
\hline & LVI-30 & $4,31 \pm 0,63$ & $4,92 \pm 1,12$ & $4,23 \pm 0,73$ & $4,31 \pm 0,48$ \\
\hline \multirow{2}{*}{ Fadiga } & Temax & $5,77 \pm 2,88$ & $18,77 \pm 7,89^{a}$ & $13,15 \pm 4,71$ ab & $10,38 \pm 3,43^{a b}$ \\
\hline & LVI-30 & $6,62 \pm 2,26$ & $8,08 \pm 2,63$ & $5,69 \pm 1,97^{b}$ & $5,92 \pm 2,56^{b}$ \\
\hline
\end{tabular}

ANOVA para medidas repetidas, com post-hoc utilizando Tukey Test. ${ }^{a}-$ diferente do basal; ${ }^{b}-$ diferente do imediatamente após. Resultados significativos para $p \leq 0,05$. Dados apresentados em média \pm desvio-padrão. Legenda: SEES- Escala Subjetiva de Experiência em Exercício; TEmax- Teste de carga progressiva até exaustão máxima; LVI-30- Teste em carga retangular na intensidade do limiar ventilatório I por 30 minutos.

$\mathrm{Na}$ tabela 5 apresentamos os resultados da escala de bem-estar para o Grupo de Ansiedade Traço Média $\left(\mathrm{G}_{\mathrm{ATM}}\right)$. Quando este grupo executou o protocolo TEmax, houve diferença apenas na dimensão fadiga em relação à condição basal, nos momentos imediatamente após $(\mathrm{p}=0,0002) ; 30 \mathrm{~min} .(\mathrm{p}=0,002)$. Houve também diferenças na dimensão fadiga em relação ao momento imediatamente após nos tempos $30 \mathrm{~min}$. ( $\mathrm{p}=0,02) ; 60 \mathrm{~min} .(\mathrm{p}=0,0002)$. No protocolo LVI-30, também houve diferença para a dimensão fadiga, no momento Imediatamente Após 
$(\mathrm{p}=0,008)$ em relação a condição Basal. Não houve diferenças significativas em nenhuma variável na comparação entre os grupos.

Tabela 5 - Resultados de bem estar (SEES) nos diferentes protocolos de exercício físico e momentos considerados para Grupo de Ansiedade Traço Média $\left(G_{\text {ATM }}\right.$.

\begin{tabular}{|c|c|c|c|c|c|}
\hline \multicolumn{2}{|c|}{ Variáveis } & \multirow{2}{*}{ Basal } & \multirow{2}{*}{$\begin{array}{l}\text { Imediatamente } \\
\text { Após }\end{array}$} & \multirow{2}{*}{30 minutos } & \multirow{2}{*}{60 minutos } \\
\hline Dimensões & Testes & & & & \\
\hline \multirow{2}{*}{$\begin{array}{l}\text { Bem-estar } \\
\text { Positivo }\end{array}$} & TEmax & $16,15 \pm 2,76$ & $16,08 \pm 4,03$ & $16,46 \pm 3,78$ & $15,46 \pm 4,14$ \\
\hline & LVI-30 & $16,85 \pm 2,73$ & $15,92 \pm 3,35$ & $15,69 \pm 3,28$ & $15,23 \pm 2,92$ \\
\hline \multirow{2}{*}{$\begin{array}{l}\text { Distresse } \\
\text { Psicológico }\end{array}$} & TEmax & $6,69 \pm 4,40$ & $8,23 \pm 4,30$ & $8,08 \pm 5,16$ & $6,54 \pm 2,90$ \\
\hline & LVI-30 & $4,62 \pm 1,04$ & $5,69 \pm 3,15$ & $4,92 \pm 2,02$ & $5,46 \pm 2,88$ \\
\hline \multirow{2}{*}{ Fadiga } & Temax & $8,92 \pm 2,84$ & $19,31 \pm 8,24^{a}$ & $14,85 \pm 6,24$ ab & $11,38 \pm 5,49^{b}$ \\
\hline & LVI-30 & $6,15 \pm 1,38$ & $10,38 \pm 5,88^{a}$ & $7,62 \pm 4,93$ & $7,54 \pm 4,18$ \\
\hline
\end{tabular}

ANOVA para medidas repetidas, com post-hoc utilizando Tukey Test. ${ }^{a}$ - diferente do basal; ${ }^{b}-$ diferente do imediatamente após. Resultados significativos para $\mathrm{p} \leq 0,05$. Dados apresentados em média \pm desvio-padrão. Legenda: SEES- Escala Subjetiva de Experiência em Exercício; TEmax- Teste de carga progressiva até exaustão máxima; LVI-30- Teste em carga retangular na intensidade do limiar ventilatório I por 30 minutos.

\section{DISCUSSÃO}

Nossos resultados sugerem de maneira inédita que independente da ansiedade traço (entre baixa e média) os jovens responderam de maneira semelhante ao exercício físico de moderada e alta intensidade em relação ao bem estar e ansiedade, sendo o exercício físico de alta intensidade mais benéfico para estes jovens com efeito ansiolítico em ambos os grupos além de proporcionar manutenção da percepção de bem-estar.

Apesar de ser uma análise intragrupo, chamamos a atenção para a percepção de fadiga destes jovens, ou seja, sujeitos mais ansiosos demonstraram menor tolerância ao exercício físico de longa duração com aumento de fadiga após o protocolo em intensidade moderada, uma vez que os grupos estudados possuiam semelhante condição física.

Neste ponto, demonstra-se uma limitação do estudo, ou seja, a necessidade de inclusão de um terceiro grupo com alto nível de ansiedade traço (extremo superior da classificação utilizada no instrumento) para comparações detalhadas do efeito ou possível influência desta nas variáveis mencionadas durante este trabalho.

Esta limitação e consequente necessidade surge ao observamos os relatos de Spielberger ${ }^{7}$ et al., em que é plausível esperar de uma maneira geral, que indivíduos com elevados níveis de ansiedade traço exibam elevações de ansiedade estado mais frequentes do que aqueles com baixos níveis de ansiedade traço, por tendência a reagir a situações como perigosas ou ameaçadoras. Estes sujeitos (alto nível de ansiedade traço) são mais propensos ao aumento de ansiedade estado em situações que envolvam relação interpessoal que representem alguma ameaça a auto-estima ou situações que envolvam possibilidade de experienciar fracasso. $\mathrm{O}$ autor ainda complementa que este fato (percepção de situações como ameaça) é influenciado por experiências passadas surgindo a necessidade de futuros estudos controlarem o envolvimento dos participantes com o exercício físico no passado em relação a competições, modalidades e tempo de prática.

O efeito ansiolítico encontrado em nosso estudo após o exercício físico intenso, foi semelhante ao estudo de Garvin ${ }^{22}$ et al., porém com protocolo de 50 minutos a 
$70 \% \mathrm{VO}_{2 \text { maxx }}$. Por outro lado, este efeito ansiolítico foi distinto ao trabalho de Kol$\operatorname{tyn}^{23}$ et al., em que jovens fisicamente ativos do sexo masculino não modificaram o estado de ansiedade após realização de protocolos de alta intensidade, de maneira constante e incremental em cicloergômetro, envolvendo exaustão física.

Já o estudo de $\mathrm{O}^{\prime} C_{\text {Connor }}^{8}$ et al., demonstrou que as respostas de ansiedade ao exercício físico máximo dependem do nível de ansiedade que o indivíduo possuía antes de começar um programa de exercício físico, bem como do tempo de recuperação após tal sessão, já que nos primeiros cinco minutos, após o exercício físico, o nível de ansiedade é elevado e diminuindo apenas quando se atinge 10 a 15 minutos pós-realização desta sessão.

Em relação a intensidade moderada, representada pelo protocolo LVI-30, a manutenção dos níveis de ansiedade observada em ambos os grupos $\left(\mathrm{G}_{\text {ATB }}\right.$ e $\left.\mathrm{G}_{\text {ATM }}\right)$ foi diferente dos resultados encontrados por Petruzzello \& Tate ${ }^{24}$ e Tate e Petruzzello $^{25}$, isto é, os autores observaram resposta ansiolítica nos dois estudos, em jovens de ambos os sexos, 30 minutos após protocolo moderado semelhante ao proposto neste estudo.

Em relação ao bem-estar, o exercício físico de 30 minutos em intensidade moderada proposto neste estudo não proporcionou alterações neste aspecto independentemente da ansiedade traço, de maneira distinta aos estudos de $\mathrm{Cox}^{26}$ et al., e Daley \& Welch ${ }^{27}$. Esses autores encontraram aumento de bem-estar após trinta minutos de exercício físico aeróbio em intensidade moderada, após trinta minutos e duas horas de execução da sessão respectivamente.

Já para o protocolo intenso, apesar do grupo $G_{\text {ATB }}$ ter apresentado aumento na dimensão distresse psicológico imediatamente após sua execução, isso não foi suficiente para dizer que houve piora no bem-estar, desta forma, pode-se afirmar que houve comportamento semelhante para os grupos $\left(\mathrm{G}_{\mathrm{ATB}}, \mathrm{G}_{\mathrm{ATM}}\right)$ estudados em relação a manutenção da sensação de bem-estar.

Este foi um achado novo e contrário ao que a literatura aponta recentemente demostrando inicialmente que acima dos diferentes tipos de metodologias empregadas, a relação entre a alta intensidade de exercício físico com o afeto necessita ainda ser melhor investigada.

O estudo de $\mathrm{Hall}^{28}$ et al., demonstrou o efeito do aumento da intensidade de exercício físico, em protocolo semelhante ao aplicado em nosso estudo, nas respostas afetivas de jovens de ambos os gêneros. Os autores concluíram que a intensidade de exercício físico envolvendo transição para o metabolismo anaeróbio resultou em impacto negativo no afeto, podendo até mesmo diminuir a adesão ao exercício físico de alta intensidade.

Esta afirmação é limitada na comparação a este estudo, pois não foi medido o afeto em momentos de transição para o metabolismo anaeróbio, apesar dos autores utilizarem o teste progressivo até a exaustão, servindo, no entanto como um indicador da relação entre o exercício físico intenso e bem-estar.

A metanálise de Ekkekakis \& Petruzello ${ }^{29}$ aponta para o fato de que aumento na intensidade parece estar associado a diminuição na positividade do afeto. Já a metanálise de Reed \& Buck ${ }^{30}$ (2009) apontou apenas para a relação entre exercício físico moderado e bem estar, ou seja, que o exercício físico aeróbio moderado parece estar relacionado a relatos de melhora no bem estar e que a magnitude desta resposta depende dos níveis desta variável pré-exercício físico.

Diante do exposto, uma nova perspectiva na relação exercício físico, ansiedade e bem estar é apresentada a literatura em relação a indivíduos com diferentes níveis de ansiedade traço e sua relação com variáveis psicobiológicas no âmbito da 
especificidade envolvendo a prescrição do exercício físico, seja ele em moderada ou alta intensidade.

Como conclusão, apesar de ser uma análise intragrupo, parece que sujeitos mais ansiosos demonstraram menor tolerância ao exercício físico de longa duração respondendo com aumento de fadiga após sessão de moderada intensidade. Portanto os resultados deste estudo sugerem de maneira inédita que independente da ansiedade traço (entre baixa ou média) os jovens responderam de maneira semelhante ao exercício físico de moderada e alta intensidade em relação ao bem estar e ansiedade, sendo o exercício físico de alta intensidade mais benéfico para estes jovens com efeito ansiolítico em ambos os grupos além de proporcionar manutenção da percepção de bem-estar.

O entendimento da variável psicobiológica ansiedade traço ampliará o campo de prescrição, por parte dos educadores físicos, para jovens fisicamente ativos, sendo que a alta intensidade aplicada de maneira progressiva torna-se uma opção atrativa para estes jovens, podendo ser utilizada na redução da ansiedade e manutenção da sensação de bem-estar sem interferir negativamente no afeto e consequentemente na adesão dos participantes a um programa de exercício físico.

\section{Contribuição dos Autores}

HKM Antunes e REEPC Miranda tiveram a ideia original e participaram de todas as fases do estudo (concepção, coleta de dados, análises até a escrita). Silva Neto E contribuiu com a coleta de dados e elaboração do material escrito. MT De Mello contribuiu com a escrita do material e colaborou com a revisão do texto. Todos os autores participaram da escrita do manuscrito e aprovaram a versão submetida para publicação.

\section{Agradecimentos; Suporte técnico e financeiro:}

Os autores agradecem: Associação Fundo de Incentivo à Pesquisa - AFIP; Centro de Estudos em Psicobiologia e Exercício - CEPE; Centro de Estudo Multidisciplinar em Sonolência e Acidentes - CEMSA; Coordenação de Aperfeiçoamento de Pessoal de Nível Superior - CAPES; FAPESP (processo no.: 2008/064433); Conselho Nacional de Desenvolvimento Científico e Tecnológico - CNPq (45074/2011-4), pelo auxílio financeiro.

\section{REFERÊNCIAS BIBLIOGRÁFICAS}

1. Antunes HK, Stella SG, Santos RF, Bueno OF, de Mello MT. Depression, anxiety and quality of life scores in seniors after an endurance exercise program. Rev Bras Psiquiatr. 2005;27(4):266-71.

2. De Mello MT, Lemos Vde A, Antunes HK, Bittencourt L, Santos-Silva R, Tufik S. Relationship between physical activity and depression and anxiety symptoms: a population study. J Affect Disord. 2013;149(1-3):241-6.

3. Chida Y, Steptoe A. Positive psychological well-being and mortality: a quantitative review of prospective observational studies. Psychosom Med. 2008;70(7):741-56.

4. Araújo SR, de Mello MT, Leite JR. Anxiety disorders and physical exercise. Rev Bras Psiquiatr. 2007;29(2):164-71.

5. Greenberg PE, Sisitsky T, Kessler RC, Finkelstein SN, Berndt ER, Davidson JR, Ballenger JC, Fyer AJ. The economic burden of anxiety disorders in the 1990s. J Clin Psychiatry. 1999;60(7):427-35.

6. Pasquini M, Berardelli I. Anxiety levels and related pharmacological drug treatment: a memorandum for the third millennium. Ann Ist Super Sanita. 2009;45(2):193-204.

7. Spielberger, C.D.; Gorshusch, R.L.; Lushene, E.; Manual for the State-Trait Anxiety Inventory (“Self-Evaluation Questionnaire”). Consulting Psychologist Press, Palo Alto, C.A, 1970. 
8. O’Connor PJ, Petruzzello SJ, Kubitz KA, Robinson TL. Anxiety responses to maximal exercise testing. Br J Sports Med. 1995;29(2):97-102.

9. Cramer SR, Nieman DC, Lee JW. The effects of moderate exercise training on psychological well-being and mood state in women. J Psychosom Res. 1991;35(4-5):437-49.

10. Acevedo E, Ekkekakis P. Affective responses to acute exercise. In: Acevedo, E.; Ekkekakis, P (org.). Psychobiology of Physical Activity. 2. Ed. Champaign: Human Kinetics. p. 91-109, 2006.

11. Tate AK, Petruzzello SJ. Varying the intensity of acute exercise: implications for changes in affect. J Sports Med Phys Fitness. 1995; 35(4):295-302.

12. Conn VS. Anxiety outcomes after physical activity interventions: meta-analysis findings. Nurs Res. 2010;59(3):224-31.

13. Samulski, D.; Noce, F.; Chagas, M.H. Estresse. In: Samulski, D.M. Psicologia do Esporte. 2.ed. Brasil: SP, p.157-190, 2002.

14. St Clair Gibson A, Baden DA, Lambert MI, Lambert EV, Harley YX, Hampson D, Russell VA, Noakes TD. The conscious perception of the sensation of fatigue. Sports Med. 2003;33(3):167-76.

15. Denadai BS, Ortiz MJ, Stell S, Mello MT. Validade de velocidade crítica para determinação dos efeitos do treinamento no limiar anaeróbio em corredores de endurance. Rev Port Cien Desp. 2003; 3(1):16-23

16. Sassi A, Marcora SM, Rampinini E, Mognoni P, Impellizzeri FM. Prediction of time to exhaustion from blood lactate response during submaximal exercise in competitive cyclists. Eur J Appl Physiol. 2006; 97(2):174-80.

17. Tanaka H, Monahan KD, Seals DR. Age-predicted maximal heart rate revisited. J Am Coll Cardiol. 2001;37(1):153-6.

18. Wasserman K. Determinants and detection of anaerobic threshold and consequences of exercise above it. Circulation. 1987;76(6 Pt 2):VI29-39.

19. Mcauley E, Courneya K. The subjective exercise experiences scale (SEES): development and preliminary validation. JSEP. 1994; 16:163-177.

20. Lox CL, Rudolph DL. The subjective exercise experiences scale (SEES): factorial validity and effects of acute exercise. J Soc Behav Pers. 1994;9(4):837-844.

21. Biaggio AMB, Natalicio L. Manual para o inventário de ansiedade traço-estado (Idate). Rio de Janeiro, Centro Editor de Psicologia Aplicada-CEPA, 1979.

22. Garvin AW, Koltyn KF, Morgan WP. Influence of acute physical activity and relaxation on state anxiety and blood lactate in untrained college males. Int J Sports Med. 1997; 18(6):470-476.

23. Koltyn KF, Lynch NA, Hill DW. Psychological Responses to Brief Exhaustive Cycling Exercise in the Morning and the Evening. Int J Sport Psychol. 1998; 29:145-156.

24. Petruzzello SJ, Tate AK. Brain activation, affect, and aerobic exercise: An examination of both state-independent and state-dependent relationships. Psychophysiology. 1997; 5:527-533.

25. Tate AK, Petruzzello SJ. Varying the intensity of acute exercise: implications for changes in affect. J Sports Med Phys Fitness. 1995; 35(4):295-302.

26. Cox RH, Thomas TR, Davis JE. Positive and negative affect associated with an acute bout of aerobic exercise. JEPonline 2001; 4:13-2001.

27. Daley AJ, Welch A. The effects of $15 \mathrm{~min}$ and $30 \mathrm{~min}$ of exercise on affective responses both during and after exercise. J Sport Sci. 2004;22(7):621-628.

28. Hall EE, Ekkekakis P, Petruzzello SJ. The affective beneficence of vigorous exercise revisited. Br J Health Psychol. 2002;7(1):47-66.

29. Ekkekakis P, Petruzzello SJ. Acute Aerobic Exercise and Affect Current Status, Problems and Prospects Regarding Dose-Response. Sports Med. 1999:28(5):337-374.

30. Reed J, Buck S. The effect of regular aerobic exercise on positive-activated affect: A metaanalysis. Psychology Sport Exerc. 2009;10:581-594.

$$
\begin{array}{r}
\text { Endereço para Correspondência } \\
\text { Profa. Dra. Hanna Karen Moreira Antunes } \\
\text { Departamento de Biociências } \\
\text { Universidade Federal de São Paulo - Cam- } \\
\text { pus Baixada Santista } \\
\text { Rua Silva Jardim, } 136 \text { - Térreo - Vila Mathi- } \\
\text { as - Santos- SP } \\
\text { CEP: 11015-020 Brasil } \\
\text { Telefone: (13) 3878-3732 } \\
\text { e-mail: hanna.karen@unifesp.br }
\end{array}
$$

Recebido 25/12/2013

Revisado 02/01/2013

Aprovado 02/01/2013 